\title{
High Science Value Return of Small Spacecraft at Mars
}

First and corresponding author:

N. Barba ${ }^{1}$, Jet Propulsion Laboratory, California Institute of Technology.

Email.nathan.j.barba@jpl.nasa.gov

Phone. 623-297-4382

Co-authors in alphabetical order: A. Austin ${ }^{1}$, D. Banfield ${ }^{2}$, A. Chmielewski ${ }^{1}$, P. Clark ${ }^{1}$, W. Coogan ${ }^{10}$, R. Conversano ${ }^{1}$, V. Cormarkovic ${ }^{1}$, S. Diniega ${ }^{1}$, C. Edwards ${ }^{1}$, R. French ${ }^{3}$, J. Fuller ${ }^{4}$, M. Gallagher ${ }^{1}$, L. Giersch $^{1}$, T. Komarek ${ }^{1}$, R. Lillis ${ }^{5}$, C. Loghry ${ }^{6}$, S. Matousek ${ }^{1}$, L. Montabone , M. Mischna ${ }^{1}$, P. Niles ${ }^{8}$, C. Norton $^{9}$, M. Shihabi ${ }^{1}$, V. Stamenković ${ }^{1}$, C. Swann ${ }^{11}$, F. Tan ${ }^{9}$, R. Woolley ${ }^{1}$.

${ }^{1}$ Jet Propulsion Laboratory, California Institute of Technology, ${ }^{2}$ Cornell University, ${ }^{3}$ RocketLab US A, ${ }^{4}$ Virgin Orbit, ${ }^{5} U C$ Berkeley, ${ }^{6}$ Moog, Inc., ${ }^{7}$ Space Science Institute, ${ }^{8} \mathrm{~N} A S$ A JSC, ${ }^{9} \mathrm{~N} A S$ A HQ ${ }^{10}$ Firefly Aerospace, ${ }^{11}$ US Naval Research Laboratory. 


\section{Introduction}

In the coming decade, small spacecraft (SSc) missions, both orbiters and landers, can provide decadalclass science, augment flagship missions, establish dedicated Mars infrastructure, and gather key reconnaissance in preparation for human exploration of Mars, at mission costs that are a fraction of the current Discovery Program cost cap.

The paradigm shift in capability cost can be attributed to many factors, including an order-ofmagnitude reduction in cost to launch, enabled by rideshare and emerging small launch vehicles, new advances in propulsive and aerocapture technologies, the existence and development of SSc-compatible science instruments capable of high-precision and/or fundamentally novel measurements enabling decadal-class science, innovative low-cost rough landers, the proliferation of commercial development and use of SSc technology, and new policies and programmatic frameworks that facilitate a future SSc program.

This paper presents a detailed framework for a science-driven SSc program that can augment existing Mars Exploration Program objectives and be a model and enabler for future SSc exploration across the Solar System. We will discuss mission concepts that are compatible with small orbiters and landers, and the instruments, technologies, and infrastructure that enable them. The timely opportunity for low-cost, frequent access to Mars is given now. This Mars opportunity can act as a springboard to reduce costs and mature technologies needed for small spacecraft missions going at a later stage to Venus, the asteroid belt, and the Jovian system.

\section{Compelling Science}

\subsection{Recent MEPAG Updates and Compatibility with SSc}

The MEPAG Goals document has undergone several changes since the last Decadal Survey; a recent modification in 2020 put extant life, the deep subsurface, polar science, and, in general, dynamic "processes" and reservoir exchange into the spotlight. As we will show below, SSc can address a variety of these new MEPAG themes and much more.

\subsection{The Right Kind of Science for SSc}

The approach that we have undertaken is to identify the science questions that can be answered costeffectively based on emerging SSc capabilities. We have found that SSc can answer questions related to various objectives of interest, including:

- Characterizing the atmosphere and ionosphere, energetic particles, local and global dust evolution, wind, temperature, pressure and electromagnetic fields on a global scale and across variable heights above the surface.

- Assessing modern-day habitability and subsurface properties by: characterizing deep subsurface liquid groundwater (a possible last modern habitat on Mars), localizing the sinks and sources or trace gases (such as methane), estimating the delivery of organics by meteorites, and constraining shallow subsurface redox profiles;

- Enabling polar science, based on the higher permitted risk for SSc to land in typical high-risk regions such as the poles; assessing resource inventories, including ice and ores;

- Determining, with high spatiotemporal resolution, current climate and its variability as a function of season, latitude, time of day, and terrain;

SSc offer unique science tied to (1) performing fundamental and new measurements, (2) executing targeted science objectives with a focused instrument suite, (3) accessing landing regions that are normally too risky, (4) enabling 3D (global) and 4D (global and temporally continuous) measurements (the latter also includes the exploration of large-scale areas with sensordriven mini-scouts). The following sections will discuss a diverse set of selected mission concepts that address these aspects (see Figure 1).

Barba 1 


\subsection{Fundamental \& New Single Measurements}

While many missions have targeted Mars, there are still unique properties of great scientific importance that have not yet been measured and which could be determined with one single instrument. Fundamentally new measurements from orbit would include the determination of exogenic material, including organics, falling onto the Martian surface, and wind measurements across extended heights above the surface. On the surface, these would include measurements of liquid groundwater to depths of several kilometers that could be achieved with SSc-compatible low-mass transient electromagnetic (TEM) sounders, electromagnetic fields on the surface driven by dust and solar wind interactions with magnetotelluric instruments, water and $\mathrm{CO}_{2}$

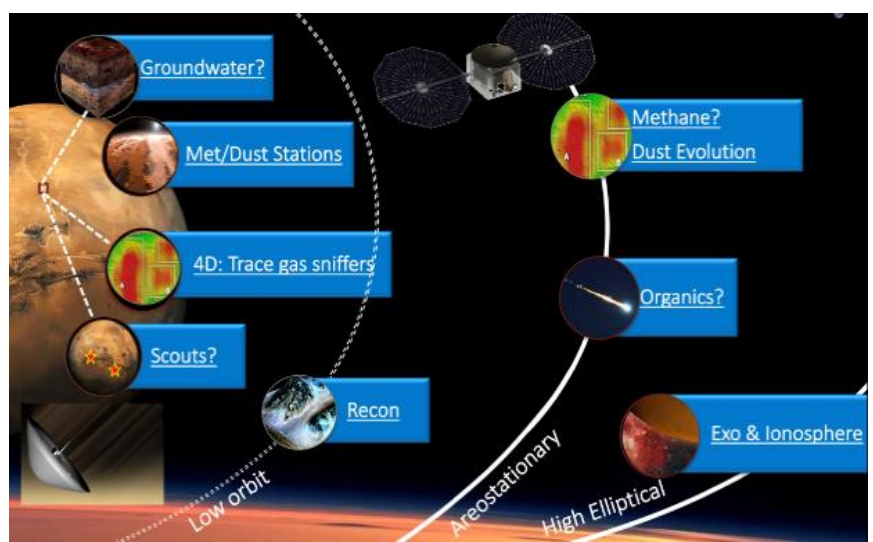

Figure 1: Illustration of science mission concepts enabled by SSc, on the surface and in orbit, as well as low-orbit reconnaissance missions for science, exploration, hazard avoidance, and telecom infrastructure purposes. condensation at the poles, or shallow subsurface redox profiles.

\subsubsection{Science: Constellations, Continuity, \& Causality}

Many processes that occur on Mars - and which are related, for example, to seismology, climate, and atmospheric erosion - can only be better understood if we enable 4D monitoring (globally in three dimensions and also across time). This would not just allow us to understand what causes, for example, dust storms or Mars quakes, or what drives water movement from the very shallow subsurface to the ionosphere, but would also allow us to set monitoring stations for human exploration-emphasizing how big Mars science questions of the future tightly link to human Mars exploration.

Of particular interest are (i) orbital and/or surface constellations/networks, (ii) science from areostationary orbit, enabling continuous observations over diurnal and seasonal scales, in support of an understanding of causality, and (iii) sensor-driven surface scouts.

Orbital constellations using SSc can generate a detailed understanding of 4D processes in the exosphere, ionosphere, atmosphere, and on the ground. Specifically, high-cadence, simultaneous measurements of the physical processes that drive matter and energy flow between and within the various climate domains, from the surface to the exosphere (globally, diurnally, and seasonally), could reveal the spatial and temporal connections governing the current Martian climate system. Deploying constellations of small satellites to carefully-chosen orbits is the best way to make the required systematic, synchronous observations of the surface, lower-middle atmosphere, thermosphere,
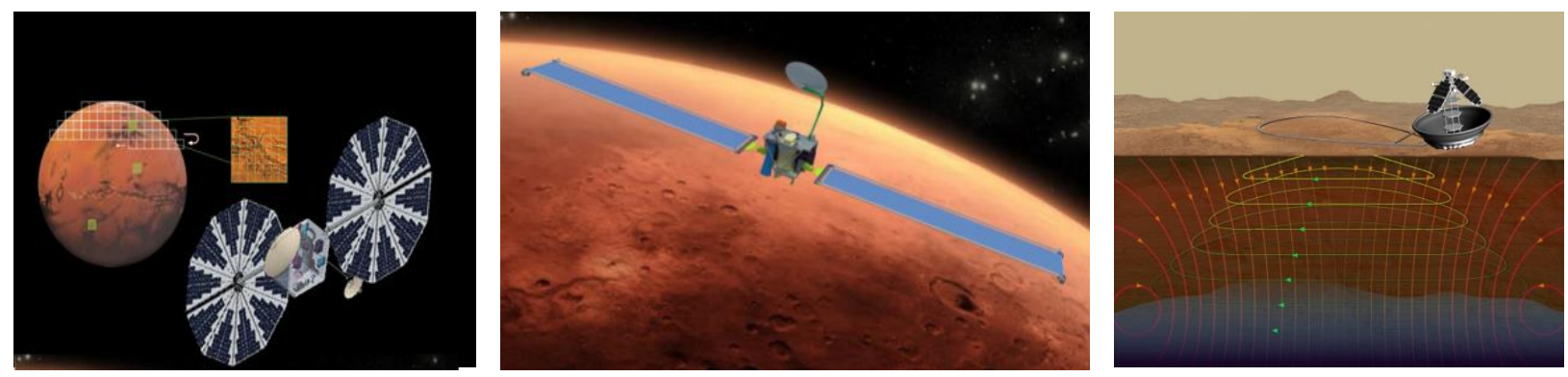

Figure 2: Mars Small Spacecraft concept portfolio from left to right: Areo Trace Gas Localizer; Mars Reconnaissance Scout; Small High Impact Energy Landing Device (SHIELD) with liquid water sounder ( $\left.\mathrm{TH}_{2} \mathrm{OR}\right)$.

Barba 2 
ionosphere, exosphere, and magnetosphere. The primary types of observation platforms necessary to make these kinds of measurements are (1) one or more areostationary orbiters at 17,000 km above the Martian surface to track sinks and sources of, for example, trace gases, as well as monitoring global weather and dust dynamics, (2) a low altitude, circular, polar orbit constellation for vertical profiling (0-100 km) of temperature, pressure, and the radiative constituents of dust, water vapor, $\mathrm{CO}_{2}$ ice, and $\mathrm{H}_{2} \mathrm{O}$ ice, (3) an elliptical, $\sim 150 \times 7000 \mathrm{~km}$ orbit pair or triplet to measure the real-time response of Mars' upper atmosphere to the highly dynamic solar wind environment to determine the spatial versus temporal variability in the Martian magnetosphere.

Surface networks could enable the characterization of climate and dust in the atmospheric boundary layer, which is generally not accessible from orbit. For identifying sources and sinks of methane, for example, surface networks might be necessary, as near-surface processes could be destroying methane at moderate heights above the surface. Surficial networks can also make use of small, high-frequency seismometers to obtain a much better picture of the global, crustal structure. Achieving mobility on the surface with SSc landers is still to be determined, but at least passive mobile assets could be compatible with a low-cost, rough landing platform—allowing surficial SSc-derived mobile scouts to explore the Martian surface and subsurface.

\subsection{Special Landing Zones Are Enabled}

Due to conventional mission risk postures, landing regions are typically limited to low latitudes, low elevations, and "landing-friendly" terrains. A low-cost SSc opportunity allows accommodation of higher risk to land in regions that are typically not accessible with normal missions, such as the polar regions, the Southern Highlands, Tharsis, and Valles Marineris, amongst others.

\subsection{Availability of Small Spacecraft High Precision Science Instruments}

A survey of domestic and international instruments bounded at $\leq 20 \mathrm{~kg}$ for orbiters and $\leq 6 \mathrm{~kg}$ for landers identified $50 \mathrm{US}$, and 35 non-US, previously flown instruments that met those criteria. There is a plethora of existing and under-development science instruments that will be compatible with Mars SSc orbiters and landers addressing a variety of science questions. Developments over the last decade continue this trend toward more compact, manufacturable (to support distributed networks), and lower mass and power instruments with tunable (e.g., wavelength, FOV) components.

\section{Low-cost, Robust Flight Architectures}

\subsection{Low Cost Architectures}

In 2018, JPL initiated a Mars small spacecraft study, which has, so far, covered a broad trade-space at both the mission and flight-system level. Three mission concepts developed in that study, demonstrating the value and viability of SSc for Mars science, are shared here: an orbiter in areostationary orbit; a low-altitude Mars orbiter, and a rough lander for the surface (see Figure 2).

A small solar electric propulsion (SEP)-powered spacecraft, beginning from Earth geostationary transfer orbit (GTO), could deliver up to $20 \mathrm{~kg}$ of science payload to an areostationary orbit for approximately half the price of a Discovery-class mission, assuming a Class D mission. Areo Trace Gas Localizer (Areo TGL) is an areostationary small orbiter capable of high temporal observation and high data throughput to support 8 gigabits of daily data volume while also simultaneously offering telecom relay link services to users on the Mars surface. As such, the flight system requires a robust high-performance telecommunication capability with MAVEN-class downlink capability and simultaneous proximity and/or cross-link connectivity with surface and ground assets.

The second orbiter architecture, named Mars Reconnaissance Scout (MRS), has a mission objective of providing a high-resolution (1 $\mu \mathrm{rad}$ Instantaneous Field of View) imagery platform for science, exploration, hazard avoidance, and infrastructure purposes. The primary instrument on the

Barba 3 
MRS orbiter concept would be a 33-cm high-resolution near field imager on an SSc orbiter in an MRO-analogous orbit. Due to the high reliability nature of an infrastructure mission, these missions were assumed to be risk class B. MRS has a total payload capacity of $42 \mathrm{~kg}$ in low Mars orbit with a Phase A-D total mission cost estimate below the Discovery cap. MRO and MRS both have a main instrument capable of $1 \mu \mathrm{rad}$ IFOV, though MRO has 3.5 times the payload mass in additional instrumentation.

The last case study examined, the VALKYRIE concept, landed up to six individual landers with their own $6 \mathrm{~kg}$ water detection instrument, $\mathrm{TH}_{2} \mathrm{OR}$, to the surface of Mars using a rough lander concept under development at JPL called SHIELD. SHIELD utilizes a low ballistic coefficient to stabilize and decelerate a landing craft to an expected entry velocity of $50 \mathrm{~m} / \mathrm{s}$, limiting landing loads to $<1000 \mathrm{~g}$. Under this assumption, on a single SHIELD rough lander, the VALKYRIE science mission concept [Edwards et al., 2020] suggests a $6 \mathrm{~kg}$ payload on a single rough lander could be delivered to the surface of Mars for \$120-200 M (Class D, A-D, without launch cost). The first option of $\sim \$ 120 \mathrm{M}$ is only valid for a fully hosted lander (carried by another dedicated mission such as MSR or the proposed Ice Mapper). The higher price target consists of a range of $\sim \$ 150-200 \mathrm{M}$ if the lander goes to Mars either as a secondary payload on an ESPA or as a primary on a dedicated launcher.

If three Class D SHIELD landers were accommodated on an independent low-cost cruise element; the deliverable payload would be 3 separate $6-\mathrm{kg}$ payloads, for a total mission cost below Discovery ( $\sim \mathbf{3 0 0}$ M A-D + launch cost). Bringing the same mission to Class B would allow closure just within the Discovery cost cap. By contrast, using an InSight-type lander and Class B would only allow delivering one groundwater sounder to one location under the Discovery Class cost cap. Please note that the total cost of $\sim \$ 300 \mathrm{M}$ for three landers facilitates a price/unit drop by $\sim \$ 50-100$ $\mathrm{M}$ - opening up opportunities for international collaboration and cost sharing.

These three examples illustrate the power of Class D SSc to enhance science return and enable costs well below Discovery Class. Several other concepts have also been explored by other groups and organizations. Each of the concepts discussed here was costed using the JPL Team X costing center, assuming the spacecraft was built at JPL.

\subsection{Low-Cost Rough Landers - SHIELD}

The Small, High Impact Energy Landing Device (SHIELD) concept is currently under development at JPL. SHIELD is a new entry, descent, and landing (EDL) technology that will enable low-cost access to the surface of Mars, allowing investigation of a range of Mars science topics including Life, Climate, Geology, and Preparing for Human Exploration. The current state of the art for Mars EDL technology is to use supersonically deployed parachutes during descent, and either a propulsion system or a propulsion and airbag system during landing; although these technologies have worked successfully for Mars landers with entry masses ranging from $600-3300 \mathrm{~kg}$, the size and complexity of these individual systems makes them difficult to adapt to low-cost landers with entry masses $\leq 100 \mathrm{~kg}$. In order to reduce the mass and cost of Mars landers, SHIELD replaces the supersonically deployed parachutes and complex landing systems with a small, subsonically-deployed drag skirt and passive impact attenuation device. The subsonic drag skirt limits the terminal velocity of SHIELD landers to $\leq 60 \mathrm{~m} / \mathrm{sec}$, and the impact attenuation device limits the landing deceleration to $\leq 1000 \mathrm{~g}$ 's.

\section{Connection to Human Exploration \& Global Collaborative Efforts}

Small missions provide an accessible and affordable approach to designing and flying spacecraft with the potential to reduce strategic knowledge gaps that would otherwise result in higher costs and risks for human missions to Mars. Beyond offering opportunities for technology demonstrations and Science/Technology/Engineering/Math (STEM) engagement, small missions can return key data for 
human exploration reconnaissance objectives such as for understanding weather patterns for safer human landings and surface operations; for characterizing rock and regolith properties for landing and traverse hazards and for civil engineering, and for validating high-priority scientific investigations that could be conducted by humans (and their robotic companions) at candidate human landing sites. Each of these reconnaissance thrusts needs more data points to build more robust models of the Martian environment so that mission planners can optimize human mission plans. Small missions are the quickest and most affordable option to collect these data.

SSc offer new approaches to advancing the Mars relay communications infrastructure, which has provided breakthrough increases in data return from Mars landers and rovers. Today's Mars relay orbiters are science orbiters with relay capabilities added as a secondary function. Small satellites offer the potential for affordable, dedicated comsat missions, with orbits optimized for relay function. For instance, prior studies [Edwards, 2016; Lock, 2016] have illustrated how small satellite concepts can deliver highly capable areostationary relay satellites to Mars orbit, offering continuous contact for surface assets as well as enabling relay service to low-altitude SSc orbiters.

Mars represents a scientific and exploration target of extremely high interest to the international space community. Currently, NASA, ESA (along with its ExoMars partner, Roscosmos), and ISRO are operating science missions at Mars, with China and the UAE having launched missions in July 2020, and ESA/Roscosmos and JAXA planning launches in 2022 and 2024, respectively. SSc offer an opportunity to significantly enhance the possibilities for additional spacefaring nations to actively enter into this community of Mars explorers - through drastically reducing the costs required to reach the Red Planet. The reduced costs also enable greater roles for academic institutions in mission implementation. The International Mars Exploration Working Group provides an effective forum for exploring strategies and partnerships for realizing this vision. Relevant strategies include establishing connections between science instrument and small spacecraft developers, establishing standards for small spacecraft instrument and launch vehicle interfaces, and advocating for, and manifesting, small spacecraft launch opportunities as hosted payloads on Mars-bound spacecraft and via rideshare opportunities.

\section{Policy/Programmatic}

Mars will always remain a strategically important target of interest given the wealth of scientific discoveries yet to be made as well its role within NASA's future directions for human exploration. Advances and capabilities enabled by small satellites opens and inspires new avenues of research toward these goals. Planning must begin now to look beyond the capabilities introduced by large landed stationery and rover missions, as well as large single-spacecraft orbiters, to increase the frequency, diversity, continuity, and range of science opportunities and subsequent knowledge accessible via a sustained presence at the Red Planet.

Enabling this strategy begins with regular access to space, learning how to accommodate various kinds of small satellite payloads on current and future heavy-lift launch vehicles. NASA Science Mission Directorate's Rideshare Policy (SPD-32) initiates this possibility explicitly, introducing ESPA rings on procured launch vehicles to utilize excess capacity when available. Capabilities like the Space Launch System (SLS) will provide such opportunities, but commercial opportunities exist already now as industry continues to develop heavy-lift vehicles for various programs of interest. Indeed, it is not unreasonable to assume that future science missions could take advantage of "Access to Mars" on test flights developed by well-known commercial organizations just as NASA has created opportunities to deploy smaller CubeSat missions to cis-lunar space as part of the SLS Exploration-1 and Exploration2 (EM-1/EM-2) future flights.

Spacecraft technology maturation and formulation of new mission architectures utilizing multiple small satellites (e.g. ESCAPADE and MarCO) are also key to the strategy allowing greater coverage

Barba 5 
and broader science questions to be posed and answered while managing risk to the overall Mars exploration science plan enabled by these systems. Flying a variety of missions to Mars, on a regular cadence, would inspire the next generation of scientific explorers while producing the knowledge and information essential to supporting related programs in human exploration. With the experience already gained, and continuing to mature, throughout government, academia, and industry, high priority Class D/C SSc missions could be proposed and executed affordably (and in a targeted way) to contribute to a balanced portfolio of traditional Class A/B Flagship missions. This integrated approach would not only grow the number of opportunities to explore Mars, but also the diversity of investigators that could participate in growing our baseline knowledge about the role of Mars as our planetary neighbor.

Mars is the one target in the solar system where the intensity of science exploration has warranted the establishment of a sustained, long-term telecommunication relay infrastructure. Multiple orbiters from both NASA and ESA are equipped with relay payloads; this relay network is currently optimized for services to landers and rovers and has greatly increased data return from the Martian surface. Evolution of the Mars relay network should also address high-rate relay services to future SSc orbiters. Such capabilities would further reduce the cost of Mars SSc missions by eliminating the need for each mission to carry its own high-rate direct-to-Earth telecom hardware; SSc missions with small proximity link payloads could achieve flagship-class data return via such relays. The presence of such a relay infrastructure would make Mars a particularly attractive target for SSc missions.

\section{Findings, Recommendations, and Conclusions}

SSc missions in the cost range of $\$ 100-300 \mathrm{M}$ represent a "sweet spot" for science return per dollar, leveraging technology trends in spacecraft and instrument miniaturization along with new lower-cost launch options to yield decadal-class science at a small fraction of Discovery mission costs. (We note that this range is above the current SIMPLEx $\$ 55 \mathrm{M}$ cost cap; based on our studies, we believe this $\$ 100-300 \mathrm{M}$ range is a more appropriate cost target for sciencerich planetary SSc missions.)

The lower end of this cost range is only feasible if science return is kept minimal, for highly elliptical orbits or flybys, and for fully hosted piggyback missions. Once lower Mars orbits, landing or travel to Mars without being hosted by another mission is taken into account, then costs grow above $\sim \$ 100$ M. It is particularly important to note that utilizing secondary (e.g., ESPA) or primary private launch opportunities would enable a much more frequent access to Mars, catalyzing small spacecraft development, and therefore reducing launch and spacecraft costs beyond the current \$100-300 M domain.

The low cost of SSc missions can enable multi-spacecraft constellations within Discovery cost caps, allowing new types of 4D investigations of the Martian system. Sending multiple spacecraft at Class D/C, while still being below a Discover-class cost cap can be an appropriate way of risk mitigation and can maximize science return per unit cost over a diverse portfolio of missions.

We recommend a number of specific actions to be taken in the coming decade to further advance the possibilities for SSc:

- Utilize commercial spacecraft suppliers to further reduce the cost and increase the robustness of interplanetary-capable flight systems, thus enhancing the frequency of missions to Mars;

- Include small spacecraft missions as augmentations to existing and future flight projects;

- Invest in mission-enabling SSc technologies, including low-cost landed architectures enabling access to the Martian surface and subsurface, a critical milestone for Mars exploration as stated by MEPAG and MASWG;

- Leverage growing international and academic partnerships to broaden access to Mars;

Barba 6 
- Continue the evolution of the Mars relay infrastructure to further support SSc mission users;

Ultimately, based on the high potential science return of SSc missions, we advocate the creation of a new programmatic framework of competed low-cost missions in the \$100-300 $\mathrm{M}$ to enable more frequent access to Mars in the era of MSR and beyond.

\section{Acknowledgements}

The information presented about future Mars mission concepts is pre-decisional and is provided for planning and discussion purposes only. The cost information contained in this document is of a budgetary and planning nature and is intended for informational purposes only. It does not constitute a commitment on the part of JPL and/or Caltech.

\section{References}

- BARBA, N. J., KOMAREK, T. A., STAMENKOVIC, V., GIERSCH, L., WOOLLEY, R. C., \& EDWARDS, C. D. (2020). EXPLORING MARS WITH SMALL SPACECRAFT ORBITERS AND LANDERS. LPI, (2326), 2874.

- barba, GIERSCH, STAMENKOVIC, CORMARKOVIC, SKLYANSKIY, KOMAREK, EDWARDS. “AFFORDABLE SURFACE AND SUBSURFACE EXPLORATION OF MARS USING SHEILD”, AGU 2019.

- barba, N., KOMAREK, T., WOOlley, R., Giersch, L., StAMENKOViĆ, V., GAllagher, M., \& EDWARDS, C. D. (2019, MARCH). MARS SMALL SPACECRAFT STUDIES: OVERVIEW. IN 2019 IEEE AEROSPACE CONFERENCE (PP. 1-10). IEEE.

- $\quad$ barba, N. J., KOMAREK, T. A., STAMEKOVIC, V., GIERSCH, L. R., WOOLLEY, R. C., \& EDWARDS, C. D. (2019). SMALL SPACECRAFT CAPABILITIES FOR MARS EXPLORATION. LPI CONTRIBUTIONS, 2089.

- LOCK, R. E., EDWARDS, C. D., NICHOLAS, A. K., WOOLLEY, R., \& BELL, D. J. (2016, MARCH). SMALL AREOSTATIONARY TELECOMMUNICATIONS ORBITER CONCEPTS FOR MARS IN THE 2020S. IN 2016 IEEE AEROSPACE CONFERENCE (PP. 1-12). IEEE.

- WOOLLEY, R., \& OLIKARA, Z. (2019, MARCH). OPTIMIZED LOW-THRUST MISSIONS FROM GTO TO MARS. IN 2019 IEEE AEROSPACE CONFERENCE (PP. 1-10). IEEE.

- C. D. EDWARDS, D. J. BELL, A. BISWAS, K. CHEUNG AND R. E. LOCK, "PROXIMITY LINK DESIGN AND PERFORMANCE OPTIONS FOR A MARS AREOSTATIONARY RELAY SATELLITE," 2016 IEEE AEROSPACE CONFERENCE, BIG SKY, MT, 2016, PP. 1-10, DOI: 10.1109/AERO.2016.7500680.

R. E. LOCK, C. D. EDWARDS, A. K. NICHOLAS, R. WOOLLEY AND D. J. BELL, "SMALL AREOSTATIONARY TELECOMMUNICATIONS ORBITER CONCEPTS FOR MARS IN THE 2020S," 2016 IEEE AEROSPACE CONFERENCE, BIG SKY, MT, 2016, PP. 1-12, DOI: 10.1109/AERO.2016.7500899.

The research was carried out at the Jet Propulsion Laboratory, California Institute of Technology, under a contract with the National Aeronautics and Space Administration (80NM0018D0004). (C2020. All rights reserved. Pre-Decisional Information - For Planning and Discussion Purposes Only. The cost information contained in this document is of a budgetary and planning nature and is intended for informational purposes only. It does not constitute a commitment on the part of JPL and/ or Caltech.

Barba 7 